\title{
PERFORMANCE OF CDMA SLOTTED ALOHA MULTIPLE ACCESS WITH MULTIUSER DETECTION
}

\author{
Sumit Roy \\ Dept. of Electrical Engineering \\ Univ. of Washington, Seattle, WA 98195
}

\author{
H.-Y. Wang \\ Technology Solutions Group \\ Duluth, GA 30097
}

\begin{abstract}
Packetized random access based on Code Division Multiple Access (CDMA) is considered. Our primary aim is to assess the impact of (uncoded) multi-user (MU) detectors on the performance of CDMA with slotted ALOHA. The impact of respective multiple packet reception capability for the matched filter and MMSE detector on delay/throughput improvements (vis-a-vis narrowband slotted ALOHA that are collision limited) is investigated.
\end{abstract}

\section{INTRODUCTION}

Next generation broadband PCS services systems are expected to accommodate heterogeneous traffic (i.e. integrated voice/data services) with enhanced burstiness. Premier candidates for multiple accessing in such networks include packetized CDMA random access methods that simultaneously exploit the well-documented benefits of code-division multiple access (CDMA) (robustness to fading and multi-user interference, soft degradation and hand-off capabilities in cellular systems) and packet switching [3].

A key advance in recent years has been the evolution of a class of advanced detector designs - known as multi user (MU) detection - that treat interfering users as structured signals (and not additive noise) and seek to jointly estimate them along with the desired signal. An important class of such MU detectors have linear complexity (in the number of users) and yield significantly superior link error rates as compared to the (single-user optimal) matched filter detector in near-far dominated scenarios, i.e., when the desired signal to interfering users' power is low. The primary objective

email: roy@ee.washington.edu. This work was completed while the authors were with the Univ. of Texas, San Antonio and supported by ARO Grant DAAH 04-96-10088 and State of Texas Adv. Technology Program Award 010115-248b of this work is to assess the impact of such MU detectors on the performance of CDMA (slotted) S-ALOHA access. The potential contribution of MU detectors towards improving the throughput/delay characteristics of CDMA S-ALOHA over a wireless link is best understood by examining the fundamental assumptions behind the celebrated throughput-offered load relation for narrowband S-ALOHA (infinite population model) $S=G e^{-G}$ where $S, G$ measured in packets/slot denotes the average number of successful packets transmitted (throughput) and the net (newly arrived plus backlogged) traffic (offered load), respectively. The above follows from key assumptions that (i) a successful packet transmission occurs with probability 1 if and only if one user attempts to transmit in a slot and (ii) whenever $K>1$ users simultaneously transmit, the probability of any successful transmission is identically zero. From a link-level perspective, assumption (i) is somewhat optimistic (assumes essentially no additive noise or equivalently, infinite SNR) while (ii) is most conservative as it negates the possibility of successful transmission in the presence of any multiple access interference (MAI). The second assumption is reasonably justified in the context of a wired local area network (LAN) bus with repeaters where the received signal powers from all transmitters at any destination is approximately identical, thereby leading to large probabilities of packet error with simple detectors that were not specifically designed for multiple access. However, even with sub-optimal detectors (such as the matched filter), multiple packets may be occasionally detected correctly at the receiver, thereby increasing throughput. In summary, the key assumptions of 'perfect collision' (when multiple users transmit) or 'perfect transmission' (when a single user transmits) are directly responsible for the $G e^{-G}$ throughput characteristic of S-ALOHA. In packet radio/wireless LAN 
networks, the variations in power of the received signal from different users (near-far effect) allow the possibility that the strongest user's packets may be detected correctly ('capture') even if $K>1$ users attempt to transmit simultaneously. The resultant throughput with capture $S_{c}=G e^{-\alpha G}, 0<\alpha<1$ (where $\alpha$ is determined by the threshold used for defining capture) showed improved throughput vis-a-vis S-ALOHA for higher $G$. Multi-user detection can be thought of generalization of the notion of capture to include multiple reception of 2 or more packets.

The analysis and performance evaluation reported here complement the recent investigations of $[5,6,7]$ on the same topic. The assumption of non-orthogonal yet slot-synchronized users is invoked as a convenient representation for the useful scenario where loss of design orthogonality occurs due to a combination of user asynchronism (on the uplink) and multipath channels. As is well-known, both these scenarios can be conceptualized as being equivalent to a synchronous system with a larger number of interfering users. Sec. II contains a self-contained summary of key results from MU detectors necessary for subsequent performance analysis. Sec. III derives the throughput of CDMA S-ALOHA over an AWGN channel.

\section{CDMA MULTIPLE-ACCESS MODEL}

Consider $K$-user packet-oriented communication with BPSK modulation using normalized (i.e., $\int_{0}^{T} s_{k}^{2}(t) d t=$ 1) signature waveforms $s_{1}(t), s_{2}(t), \cdots, s_{K}(t)$ over an additive white Gaussian noise channel. The received signal at the receiver input is $r(t)=S(t)+\sigma n(t)$ where $n(t)$ is white Gaussian noise with unit power spectral density. $S(t)$ is the superposition of the data signals of $K$ synchronous users, given by

$$
S(t)=\sum_{k=1}^{K} A_{k} \sum_{i=1}^{L_{p}} b_{k}(i) s_{k}(t-i T)
$$

where $A_{k}$ is received amplitude of the $k$ th user, $L_{p}$ is the packet length (assumed identical for all users), $b_{k}(i)$ is the $i$ th symbol of the $k$ th user (assumed to be binary, \pm 1 ), $\tau_{k}$ is the relative delay of the $k$ th user, $T$ is bit interval and $\sigma$ is standard deviation of the additive Gaussian noise.

The sampled output of a filter matched to $s_{k}(t)$ in the $i$ th data interval $y_{k}(i)=\int_{i T}^{(i+1) T} s_{k}(t-i T) r(t) d t, k=$ $1, \cdots, K$ yields the $K$-vector (using $i=0$ without loss

$$
\text { of generality) } \begin{aligned}
\mathbf{y}= & {\left[y_{1}, \ldots, y_{K}\right]^{T} } \\
& \mathbf{y}=\mathbf{R A} \mathbf{b}+\sigma \tilde{\mathbf{n}}
\end{aligned}
$$

where $\mathbf{R}$ is the normalized crosscorrelation matrix of the $K$ signature waveforms, $\mathbf{R}_{k, i}=\rho_{k i} \triangleq\left\langle s_{k}, s_{i}\right\rangle$ where \langle\rangle denotes the standard Euclidean inner product. $\mathbf{A}=\operatorname{diag}\left\{A_{1}, A_{2}, \cdots, A_{K}\right\} ; \mathbf{b}=\left[b_{1} \ldots b_{K}\right]^{T}$, and $\tilde{\mathbf{n}}=\int_{i T}^{(i+1) T} \mathbf{s}(t-i T) n(t) d t$. Thus $\tilde{\mathbf{n}}$ is a K-dim. Normal random vector, i.e. $\tilde{\mathbf{n}} \sim N_{K}(\mathbf{0}, \mathbf{R})$, independent of $\mathbf{b}$.

\section{Linear Multiuser Detectors}

Linear MU detectors arrive at bit decisions based on $\hat{b}_{k}=\operatorname{sgn}\left((\mathbf{L} \mathbf{y})_{k}\right)$ where $\mathbf{L}$ is a linear operator. We will use the matched filter $(\mathbf{L}=\mathbf{I})$ as a baseline, the MU receiver considered is the MMSE detector $(\mathbf{L}=(\mathbf{R}+$ $\left.\sigma^{2} \mathbf{A}^{-2}\right)^{-1}$ ) Consider

$z_{k}=\frac{(\mathbf{L y})_{k}}{B_{k k}}=b_{k}+\sum_{i \in\{1, \cdots, K\}, i \neq k} \beta_{k, i} b_{i}+\frac{\sigma}{B_{k k}}(\mathbf{L} \tilde{\mathbf{n}})_{k}$

where $\beta_{k i}=\frac{B_{k i}}{B_{k k}}, \quad B_{k i}=A_{i}(\mathbf{L R})_{k, i}, \quad i \in\{1, \cdots, K\}$

From [1], the exact bit error rate (BER) for the desired user can be written as

$$
P_{e}(m m s e)=P\left(\sum_{i \neq k} \beta_{k, i} b_{i}+\frac{\sigma}{B_{k k}}(\mathbf{L} \tilde{\mathbf{n}})_{k}>1\right)
$$

A Gaussian approximation (GA) to the above expression is given by

$\hat{P}_{e}(m m s e)=Q\left(\left(\frac{\sigma^{2}(\mathbf{L R L})_{k, k}}{A_{k}^{2}(\mathbf{L R})_{k, k}^{2}}+\sum_{i=1, i \neq k}^{K} \beta_{k i}^{2}\right)^{-1 / 2}\right)$

and has been shown to be excellent for the MMSE detector [1] over a wide range of parameters. We remark that for the matched filter, the GA is known to be problematic in the region of high SNR and large MAI [1] and should be invoked (as below) with care for regions that exclude the above. To further simplify matters, we invoke two additional assumptions as in [1]

- A1 Ideal Power control - The MU detector employs ideal power control such that the signal energies at the receiver input are identical;

- A2 Equicorrelated spreading code - The synchronous crosscorrelation $\rho_{k i}$ between any two user's codes is identically equal to $\rho$, i.e., independent of the user's codes. 
a) Conventional Detector: $\mathbf{L}=\mathbf{I}, A_{k}=A, \rho_{k i}=\rho$

Using the above, (5) becomes

$$
\hat{P}_{e}(m f)=Q\left(\left(\frac{1}{S N R}+(K-1) \rho^{2}\right)^{-1 / 2}\right)
$$

where $S N R \triangleq A^{2} / \sigma^{2}$. Note that the expressions are independent of $k$ (i.e., the BER is user independent) due to the 'symmetrizing' assumptions of perfect power control and equi-correlated spreading codes, and hence the user superscript has been dropped.

b) MMSE Detector: $\mathbf{L}=\left(\mathbf{R}+\sigma^{2} \mathbf{A}^{-2}\right)^{-1}, A_{k}=$ $A, \rho_{k i}=\rho$

Since $\mathbf{L R}=\mathbf{L}\left(\mathbf{R}+\sigma^{2} \mathbf{A}^{-2}-\sigma^{2} \mathbf{A}^{-2}\right)=\mathbf{I}-\sigma^{2} \mathbf{L} \mathbf{A}^{-2}$, we have $(\mathbf{L R})_{k, k}=1-\frac{\sigma^{2}}{A_{k}^{2}} L_{k, k}, \quad(\mathbf{L R})_{k, i}=-\frac{\sigma^{2}}{A_{i}^{2}} L_{k, i} i \neq$ $k$. Also, $\mathbf{L R L}=\mathbf{L}-\sigma^{2} \mathbf{L} \mathbf{A}^{-2} \mathbf{L}$, and hence

$$
\begin{aligned}
&(\mathbf{L R L})_{k, k}=L_{k, k}-\sigma^{2} \sum_{i=1}^{K} \frac{L_{k, i}^{2}}{A_{i}^{2}} \\
&=L_{k, k}(\mathbf{L R})_{k, k}-\sum_{i=1, i \neq k}^{K} \frac{(\mathbf{L R})_{k, i}^{2} A_{i}^{2}}{\sigma^{2}}, \\
& \frac{\sigma^{2}}{A_{k}^{2}} \cdot \frac{(\mathbf{L R})_{k, k}}{(\mathbf{L R})_{k, k}^{2}}+\sum_{i \neq k}^{K} \beta_{k i}^{2} \\
&=\frac{\sigma^{2}}{A_{k}^{2}} \cdot \frac{L_{k, k}}{(\mathbf{L R})_{k, k}}-\sum_{i \neq k}^{K} \frac{(\mathbf{L R})_{k, i}^{2} A_{i}^{2}}{(\mathbf{L R})_{k, k}^{2} A_{k}^{2}}+\sum_{i \neq k}^{K} \beta_{k i}^{2} \\
&=\frac{\sigma^{2}}{A_{k}^{2}} \frac{L_{k, k}}{(\mathbf{L R})_{k, k}}
\end{aligned}
$$

Using the above, the Gaussian approximation for MMSE detector simplifies to

$\hat{P}_{e}(m m s e)=Q\left(\frac{A_{k}}{\sigma} \sqrt{\frac{(\mathbf{L R})_{k, k}}{L_{k, k}}}\right)=Q\left(\sqrt{\frac{A_{k}^{2}}{\sigma^{2} L_{k, k}}-1}\right)$

With (A1, A2), the diagonal elements

$$
L_{k, k}=\left(1+\frac{\sigma^{2}}{A^{2}}-\frac{\rho^{2}(K-1)}{1+\frac{\sigma^{2}}{A^{2}}+(K-2) \rho}\right)^{-1}
$$

Substituting (8) into (7), the GA for the probability of bit error for MMSE detector becomes

$$
\hat{P}_{e}(m m s e)=Q\left(\frac{A}{\sigma} \sqrt{1-\frac{\rho^{2}(K-1)}{1+\frac{\sigma^{2}}{A^{2}}+(K-2) \rho}}\right)
$$

Note that as $\sigma \rightarrow 0, \hat{P}_{e}(m m s e) \rightarrow Q\left(\frac{A}{\sigma} \sqrt{1-\rho}\right)$ rapidly for $K \rho>>1$.

\section{CDMA S-ALOHA IN AWGN CHANNEL}

In a CDMA uplink, users are assumed to communicate with each other synchronously but are otherwise uncoordinated with respect to channel access. In contrast to narrowband S-ALOHA, due to CDMA and the nature of the MU receiver employed, multiple users' packets may be transmitted successfully in a single packet interval. As is customary for S-ALOHA, unsuccessful packets enter the user's buffer and are queued (along with any newly generated packet arrivals) for retransmission in the next slot interval.

\section{Throughput-Delay Analysis}

We assume that the number of users $N$ in a system is large (effectively infinite) and the probability of a user transmitting (either due to new or backlogged packets) in an interval $p$ is small such that the product $N p$ is a constant, implying that the net offered traffic is well approximated by the Poisson distribution with mean $G=N p$, i.e., the probability that exactly $K$ users transmit in in a slot is

$$
P[K]=\frac{G^{K} e^{--G}}{K !}
$$

Following [2], the average throughput of CDMA slotted ALOHA system can be written as $S=E_{K} E(S \mid K)$ where $E(S \mid K)$ is the throughput conditioned on $K$ attempted transmissions (active users) in a slot. The assumptions (A1, A2) of equi power users with equicorrelated spreading codes implies that when $K$ users transmit simultaneously, the number of successful packets is Binomial with parameters $\left(K, P_{s}\right)$, where $P_{s}$, the probability of a successful packet transmission is identical for all users. Since for the Binomial distribution $E(S \mid K)=K P_{s}$,

$$
S=\sum_{K} K P_{s}\left(K, S N R, \rho, L_{p}\right) P[K]
$$

where $P[K]$ is given by (10). For a packet consisting of $L_{p}$ data bits with no error control coding, the packet success probability $P_{s}=\left(1-P_{e}\right)^{L_{p}}$ is obtained by substituting (6) or (9) inthe above, and the throughput $S$ is numerically evaluated from (11). The results from numerical evaluation is verified by bit-level simulation of the S-ALOHA protocol that uses the above BER expresssions as inputs.

While $P_{s}$ increases with $S N R$ and decreases with $\rho$ and $L_{p}$ for both conventional and MMSE detectors, 
as expected, the sensitivity of $P_{s}$ to $K$ (number of active users) is much less for the MMSE detector than the matched filter. This is in accordance with the fact that $P_{e}$ for MMSE detector converges to a constant for sufficiently large $K$ for given $S N R$ and $\rho$. This fact has important implications for the throughput of the MMSE detector, since in (11), we may approximate $P_{s}\left(K, S N R, \rho, L_{p}\right)$ to be independent of $K$ (while this is strictly true only for $K$ sufficiently large, the variations of $P_{s}$ for small $K$ has negligible effect as verified by simulations) leading to

$$
S=\sum_{K=1}^{\infty} K P_{s} P[K] \approx P_{s}\left(S N R, \rho, L_{p}\right) G
$$

Thus the throughput $S$ is a linear function of the offered load $G$ with slope $P_{s}$, which is determined by $S N R, \rho$ and $L_{p}$.

Throughput vs offered load curves for conventional and MMSE receiver are plotted in Figs. 2-3 as function of $L_{p}, \rho$ and SNR, respectively. First, we note that for the matched filter, the $S-G$ curve is qualitatively similar to $G e^{-G}$ for narrowband S-ALOHA. Nevertheless, the normalized peak throughput $S / N$ (where $N=\frac{1}{\rho}$ is the processing gain, assuming that the user codes are $\mathrm{M}$-sequences) for the matched filter can exceed $e^{-1}=0.36$ (as for the $\rho=0.05$ curve in Fig.2) attesting to some (but limited) multiple packet reception capability - this is insufficient to overcome the innerent bottlenecks (at high $G$ ) of the S-ALOHA protocol. Also, the peak throughput for the matched filter is very sensitive to SNR, $\rho$ and $L_{p}$. In contrast, the throughput of the MMSE detector is linear with $G$ and outperforms the conventional detector for all $G$. Note in Fig. 3 that for $\mathrm{SNR}=12 \mathrm{~dB}$, the slope of the MMSE detector throughput is very nearly equal to unity, which is the theoretical maximum.

To obtain the average delay for a given test packet, the average probability of a successful packet transmission in a slot as

$$
\overline{P_{s}}=\sum_{K=0}^{\infty} P_{s}(K) P[K-1]
$$

Note that the averaging over $K$ allows for dynamic variations in the number of packet transmissions. The probability that a packet is successfully transmitted after $d \in\{1,2, \cdots\}$ attempts ( $d-1$ unsuccessful transmissions followed by a successful transmission) is the well-known geometric distribution

$$
P_{D}(d)=\left(1-\overline{P_{s}}\right)^{d-1} \overline{P_{s}}
$$

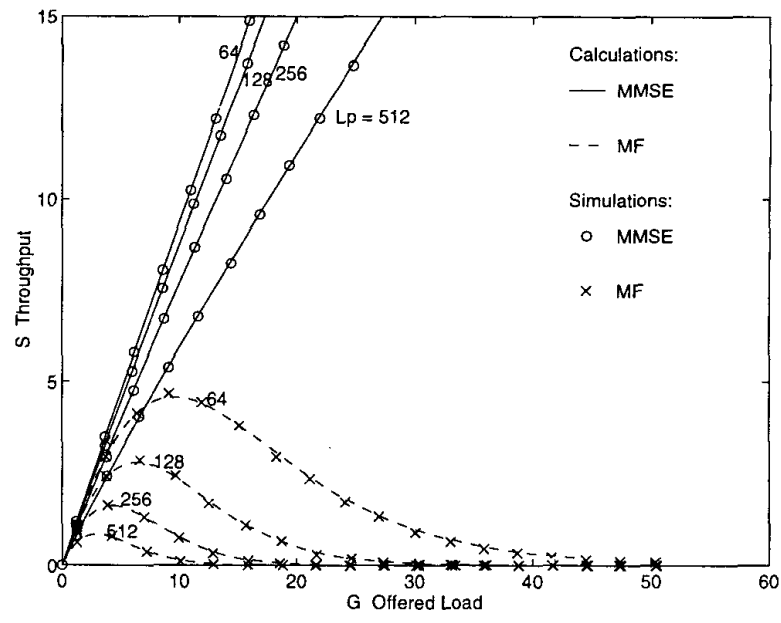

Figure 1: AWGN Channel. SNR $=10 \mathrm{~dB} . o=0.1$.

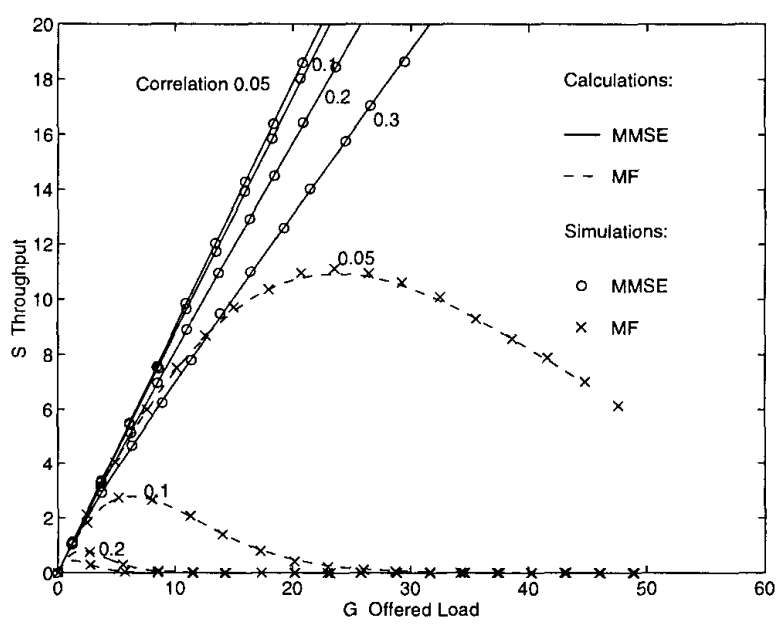

Figure 2: AWGN Channel. SNR $=10 \mathrm{~dB}, L_{n}=128$.

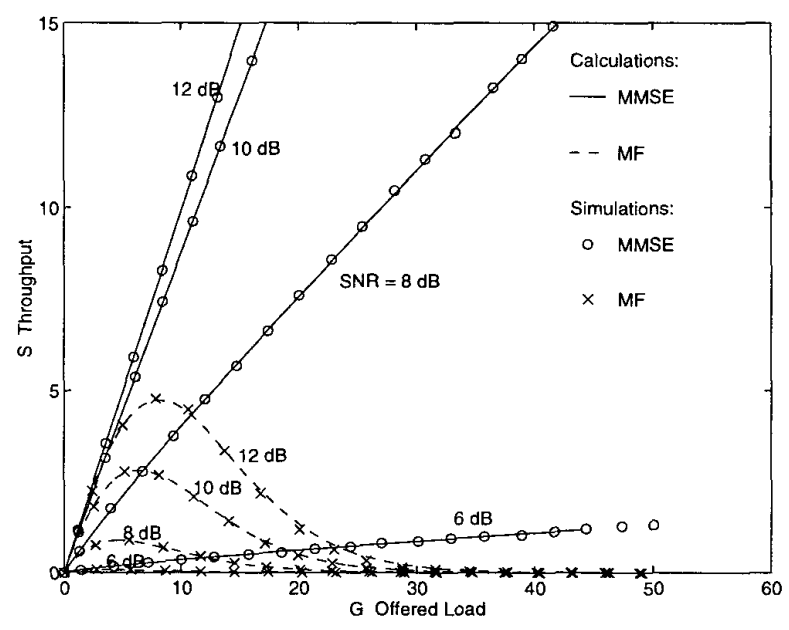

Figure 3: AWGN Channel, $\rho=0.1, L_{p}=128$. 
from which the average delay (number of transmissions prior to success) $\bar{D}$ is given by $\bar{D}=\bar{P}_{s}^{-1}$. Note that for the MMSE detector, since $P_{s}(K)$ is approximately independent of $K$ over the range of interest in $G$, it follows that $\overline{P_{s}} \approx P_{s} \sum_{K=0}^{\infty} P[K]=P_{s}$ yielding $D={\overline{P_{s}}}^{-1} \approx P_{s}^{-1}$. The results in Figs. 4,5 show that while the matched filter delay again qualitatively conforms to that of narrowband S-ALOHA $\left(e^{G}\right)$, that for the MMSE detector is approximately constant.

\section{REFERENCES}

[1] H. V. Poor, and S. Verdú, "Probability of Error in MMSE Multiuser Detection," IEEE Trans. Inform. Theory, vol 43, pp. 858-871, May, 1997.

[2] D. Raychaudhuri, "Performance Analysis of Random Access Packet-switched Code Division Multiple Access Systems," IEEE Trans. Commun. Vol. Com-29, pp. 895-901, June 1981.

[3] R. Ganesh et al., "Performance of cellular packet CDMA in an integrated voice/data network," Int. J. of Wireless Inform. Networks, vol. 1, no. 3, pp. 199-221, 1994.

[4] H.-Y. Wang, "Performance Analysis of Aloha Multiple Access with Multi-user Detection," M.S. Thesis, Div. of Engineering, Univ. of Texas at San Antonio, Dec. 1998.

[5] P. Rapajic, "Performance Analysis of Slotted ALOHA/CDMA System with Adaptive MMSE Receivers," IEICE Trans. Fund., vol. E80-A, Dec. 1997, pp. 2485-2492.

[6] M. Saito et al., "Throughput Improvement of CDMA Slotted Aloha Systems," IEICE Trans. Commun., vol. E80-B, Jan. 1997, pp. 74-79.

[7] Ari Hottinen et al., "On the Throughput of ALOHA in the presence of Multiuser Detection," Proc. IEEE Int. Conf. on Personal Wirless Communications, Mumbai, India, pp. 444-448.

[8] P. W. de Graaf et al, "Performance Comparison of a Slotted ALOHA DS/SSMA Network and a Multichannel Narrow-Band Slotted ALOHA Network," IEEE Trans. Comm., vol. 46, Apr. 1998, pp. 544-552.

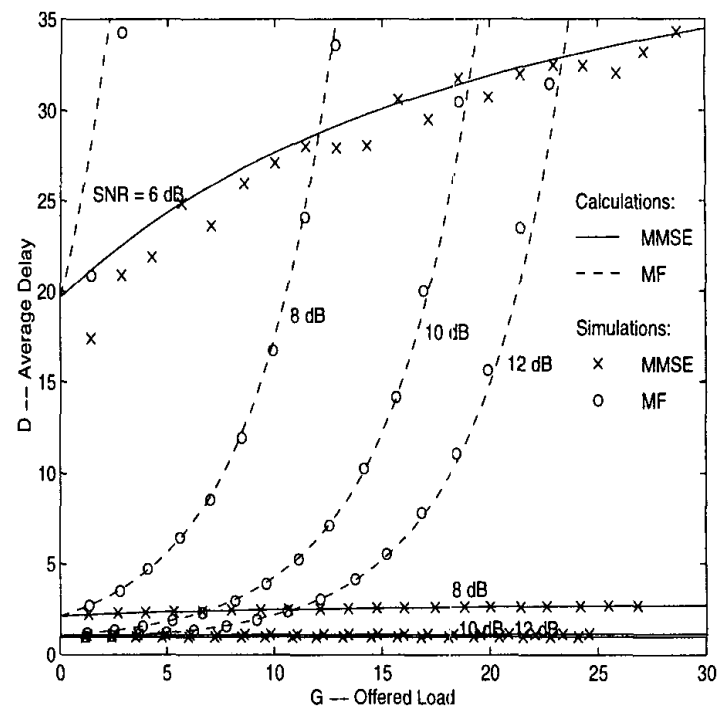

Figure 4: AWGN Channel, $\rho=0.1, L_{p}=128 \mathrm{~dB}$.

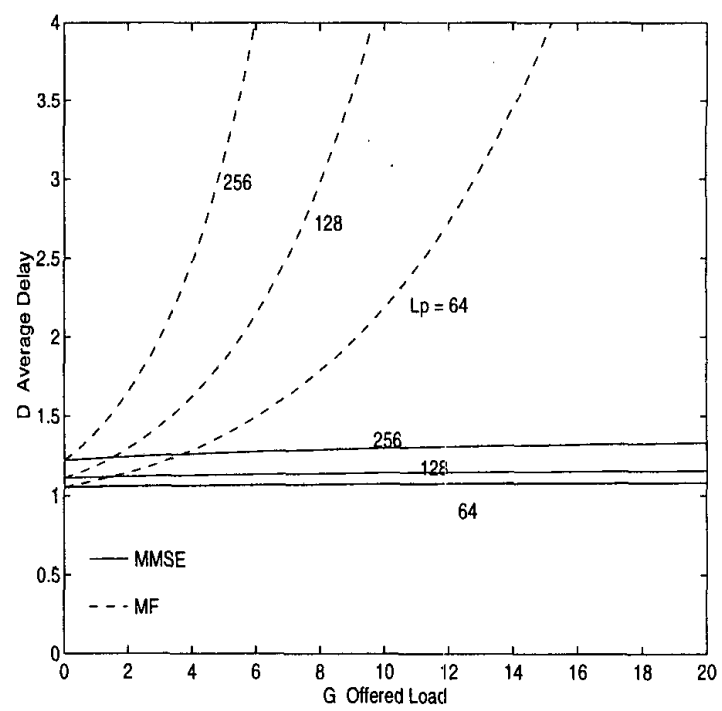

Figure 5: AWGN Channel, $\rho=0.1, S N R=10 \mathrm{~dB}$. 